\title{
Clinical Implication of Genetic Testing for Hypertrophic Cardiomyopathy
}

\section{Kazuo Komamura*}

Adjunct Assistant Professor, Cardiovascular Division, Hyogo College of Medicine, Japan

\begin{abstract}
Hypertrophic cardiomyopathy $(\mathrm{HCM})$ is caused by a dominant mutation of the sarcomere protein gene in approximately $60 \%$ of cases. Genetic testing of HCM has several benefits. Diagnosis can be confirmed if the sarcomere protein gene mutation is identified. Genetic testing enables to follow-up blood relatives who are mutationpositive only, and can identify asymptomatic and non-onset patients. Relatives with negative results of genetic testing could be freed from anguish. On the other hand, genetic testing has some disadvantages. Treatment often does not change after testing. Forty percent of cases has unknown causative genes. Relationship between gene mutation and clinical findings or treatment responsiveness is little known, and so on.

Many issues need to be resolved to incorporate genetic diagnosis into daily practice for HCM. However, genetic testing for HCM cannot be regarded as unnecessary and expensive debauchery, but rather represents a valuable measure in the medical facilities. Although there still remain questions about genetic diagnosis of $\mathrm{HCM}$, accumulated knowledge to date is fairly enough to establish the genetic testing as a useful tool for individualized management of HCM patients and their families.
\end{abstract}

Keywords: Hypertrophic cardiomyopathy; Sarcomere gene; Mutation, Genetic testing; Genetic counseling

\section{Introduction}

Hypertrophic cardiomyopathy (HCM) with left ventricular hypertrophy of unknown cause accounts for approximately one third of sudden cardiac deaths in young athletes [1]. We believe that sudden death in young people due to this disease could be reduced if a system were to be established to genetically test patients and blood relatives to make diagnosis prior to the onset of symptoms.

\section{Outline of Genetic Analysis of Hypertrophic Cardiomyopathy}

Hypertrophic cardiomyopathy is caused by a dominant mutation of the sarcomere protein gene in approximately $60 \%$ of cases [2]. At present there have been over 1,000 different types of gene mutations reported. In Japan the comprehensive sequencing of the sarcomere protein gene excluding titin has been analyzed in patients with HCM, revealing a high incidence of MTBPC3, MTH7 and TNNT2 [3]. It has been reported that some sarcomere protein gene mutations correlate with severe hypertrophy, localization, age at onset, clinical symptoms and clinical progress. For example, TNNT2 mutations are often associated with sudden cardiac death despite mild ventricular hypertrophy [4,5]. ACTC Glu101Lys mutations are common in patients with thickening at the apex of the heart [6]. Reports from the USA indicate that MYBC3 gene mutations are common in elderly onset of HCM [1]. Approximately 10\% of patients with HCM develop heart failure or a shift to the dilated phase and many will require heart transplant due to poor prognosis [5]. These have been reported 'malignant mutation'.

\section{The Expected Benefits of Genetic Testing for Hypertrophic Cardiomyopathy}

Genetic testing is expected to have the following benefits for patients suspected of having HCM.

1) To clinically diagnose HCM, ventricular wall thickening should be confirmed and other diseases that lead to hypertrophy excluded, however diagnosis can be confirmed if the sarcomere protein gene mutation, which has previously not been genetically tested, can be identified. Moreover, it is possible to predict disease severity to some extent, and in the event of a mutation suspected of being associated with prognosis of severe condition, the follow-up interval may be reduced from, for example, 1 year to 6 months.

2) If the genetic testing of index cases is positive then genetic testing of blood relatives may proceed. Asymptomatic and non-onset patients may also be identified. Of blood relatives, testing may enable follow-up to be initiated for those who are mutation-positive only. The first symptom of HCM may be sudden death during exercise, so further efforts can be made such as instructing mutation-positive individuals to lead a lifestyle that avoids excessive exercise, and taking other measures (frequency of follow-up and early intervention).

3) At present blood relatives continue to undergo echocardiography once a year while having vague concerns that they 'may be candidates for disease onset'. If such relatives were found to be clearly negative by genetic testing they would be freed from this ungrounded mental anguish, and avoid unnecessary examination or testing. For instance if there were 100,000 index cases, since it is an autosomal dominant disorder then theoretically $50 \%$ of the blood relatives would be mutation negative, so there would be 50,000 individuals identified as having sarcomere mutation and if there were 4 blood relatives per index case then there would be 200,000 blood relatives, or in other words every year 100,000 individuals are unnecessarily examined and echocardiographically tested. Utilizing genetic testing would avoid unnecessary medical expenditure.

*Corresponding author: Kazuo Komamura, Adjunct Assistant Professor, Cardiovascular Division, Hyogo College of Medicine, Japan, E-mail: kazuokomamura@gmail.com

Received August 17, 2013; Accepted September 20, 2013; Published September 25, 2013

Citation: Komamura K (2013) Clinical Implication of Genetic Testing for Hypertrophic Cardiomyopathy. Human Genet Embryol 3: 114. doi:10.4172/21610436.1000114

Copyright: (c) 2013 Komamura K. This is an open-access article distributed under the terms of the Creative Commons Attribution License, which permits unrestricted use, distribution, and reproduction in any medium, provided the original author and source are credited. 
Moreover, the '2011ACCF/ AHA guidelines for the diagnosis and treatment of hypertrophic cardiomyopathy' specifies 'evaluation of familial inheritance and genetic counseling is recommended as part of the assessment of patients with HCM (class I)', 'clinical screening (with or without genetic testing) is recommended for first-degree blood relatives of patients with HCM (class I)', 'genetic testing is reasonable in index patients to facilitate the identification of first-degree blood relatives at risk of developing HCM (class IIa)' [7].

\section{Disadvantages and Issues to be Solved in Genetic Testing for Hypertrophic Cardiomyopathy}

1) Current treatment does not change after a patient has been deemed mutation positive.

Several reports indicate a correlation between clinical findings and the causative mutation of HCM, however, they do not necessarily apply to each case. Furthermore single nucleotide polymorphisms (SNP) in addition to causative mutations are utilized as genetic modifiers, and since clinical findings may vary, a basic database for medical treatment is lacking. Is genetic testing essential to diagnose the present disease and decide on the course of treatment? From the perspective of personalized medicine effective evaluations are indispensable [8]. An appropriate evaluation index needs to be established to facilitate clinical research and obtain evidence to elucidate the relationship between clinical findings and mutations, to find the mutation correlating to effective medications (such as beta-blocker), and to prove the effectiveness of early intervention for mutation-positive individuals. Establishing genetic testing as part of general medical practice may rapidly increase the number of patients undergoing genetic testing and may enable more comprehensive clinical findings of mutations and better analysis of responsiveness to treatment.

2)Sarcomeric gene mutation is detected in approximately $60 \%$ of patients, while in the other $40 \%$ the causative gene remains unknown.

Even if limited to the sarcomeric gene, the causative gene catalogue is undeveloped. Therefore, when the previously reported gene mutation is not detected by genetic testing, the possibility remains that an unidentified gene is the cause. A cardiomyopathy registry should be established to facilitate a project in which comprehensive analyses are conducted using next-generation sequencing to create a list of genes to be tested. Furthermore, we would like to create a structure to register data in a mutation database that is consolidated in one place. At present, when unidentified mutations are detected for the first time, it is not rare that the causative mutation cannot be determined and cosegregation cannot be investigated due to a small family tree, sporadic cases and low penetration rate. In order to avoid this, a disease registry and mutation database with many cases is necessary.

\section{3)Private mutations are common}

In HCM, many mutations have low penetrance and the relationship between gene mutation and clinical findings/ treatment responsiveness is often unknown. As mentioned earlier, creating a mutation databases that can be integrated into a disease registry (i.e. registration of clinical findings and treatment responsiveness) and accumulating many various cases is important to facilitate examination of the relationship between mutations and clinical findings/ treatment responsiveness.

4) Previous reports describe many various mutations (over 1,000 types) detected in the sarcomeric gene. The workload involved in just exploring previously reported gene mutations would be enormous. Since it takes time to render results it is unlikely to be used as an auxiliary diagnostic tool.

If genetic testing were to be conducted as part of general medical practice, it would have to be ensured that analysis results be rendered quickly. Therefore efforts should be made such as developing analysis methods that allow for rapid processing, establishing a means of testing that ensures accuracy, and accumulating on specimens.

5)At present the work flow (i.e. obtaining consent, collecting and processing samples, explaining results and genetic counseling) is yet to be developed.

Currently, only a few medical institutions conduct genetic testing for HCM. Since a limited number of specialists obtain consent, are in charge of processing specimens, interpret the results, explain results to the patients and provide genetic counseling, they are extremely busy and cannot handle a greater number of patients. It would be practical to establish a center for medical treatment and build a system where patients could be referred to.

6)If genetic testing were to be widely conducted as part of general medical practice, it is expected that the number of individuals subject to testing would rapidly increase along with the high incidence of the disease, however this increase will not be able to be managed in terms of cost and labor under the current situations in the society.

If genetic testing were to be included in general medical practice then the cost will have to be a incurred as 'diagnosis expense'. If a diagnostic center that ensures accuracy were to be established, in which effective analysis could be conducted that accumulate samples then the labor burden could be reduced. There should be institutions authorized to conducted genetic testing, which should maintain the number of analysis specimens above a certain level and strive to guarantee accuracy. In the USA, genetic testing in medical care needs to be authorized by the CLIA (Clinical Laboratory Improvements Amendments). If ordered via a medical geneticist, irrelevant test requests are reduced and the validity can be ensured. It would be efficient to create a work flow in which specialized hospitals for advanced treatment are designated as the medical care center where referred patients are received, and that conduct specialist out-patient examinations, genetic counseling, obtain consent, collect samples, and request analysis to a specialized analysis center. A system should also be created that facilitates the interpretation of data at the time of rendering analysis results and the use of the results by general physicians. Laws also need to be developed so that genetic discrimination does not occur.

Furthermore, we need to deal with the following questions: 'to what extent is genetic testing technologically possible in clinical settings and not in research laboratories?', 'can the current work flow be sustained?', 'can genetic testing change decision making in medical practice?', 'what is the cost-effectiveness ratio?', 'Is there any problem with the protection of personal information and avoiding genetic discrimination?', 'Is it possible to solve problems related to the creation of a database?', and 'has the general public been educated and has a consensus of society as a whole been reached?' [9].

As shown here, many issues need to be resolved in order to incorporate genetic diagnosis as part of daily general practice for HCM. However if it were to be put in practice, not only would it be demonstrated as an auxiliary diagnostic tool for HCM, but the number of patients subject to genetic testing would rapidly increase, and it would facilitate the creation of a full catalogue of incomplete genetic mutations, elucidate the relationship between mutations and clinical findings/treatment responsiveness, and enable us to perceive a true 
Citation: Komamura K (2013) Clinical Implication of Genetic Testing for Hypertrophic Cardiomyopathy. Human Genet Embryol 3: 114. doi:10.4172/21610436.1000114

Page 3 of 3

picture of HCM in the real world.

Finally, I'd like to emphasize that the identification of a genotypepositive status remains very important in HCM, because of obvious implications for disease transmission to the offspring, deserving appropriate counseling, as well as implementation of follow-up. Furthermore, young phenotype-negative individuals represent ideal candidates for forthcoming pharmacological trials aiming at preventing phenotype development.

\section{Conclusions}

Genetic testing for HCM cannot be regarded as an unnecessary and expensive debauchery, but rather represents a valuable measure in the medical facilities. While many questions about genetic diagnosis and treatment of HCM still remain to be clarified, calling for innovations in translational research, what has been achieved so far is sufficient to establish the role of genetic testing in individualized management of HCM patients and their families. To undervalue the potential of genetic testing for HCM is a loss of very important opportunity for improving medical practice and services for cardiomyopathy.

\section{References:}

1. Maron BJ (2003) Sudden death in young athletes. N Engl J Med 349: 10641075.

2. Spirito P, Seidman CE, McKenna WJ, Maron BJ (1997) The management of hypertrophic cardiomyopathy. N Engl J Med 336: 775-785.

3. Otsuka H, Arimura T, Abe T, Kawai H, Aizawa Y, et al. (2012) Prevalence and distribution of sarcomeric gene mutations in Japanese patients with familial hypertrophic cardiomyopathy. Circ J 76: 453-461.

4. Komamura K, Iwai N, Kokame K, Yasumura Y, Kim J, et al. (2004) The role of a common TNNT2 polymorphism in cardiac hypertrophy. J Hum Genet 49: 129-33.

5. Watkins H, McKenna WJ, Thierfelder L, Suk HJ, Anan R, et al. (1995) Mutations in the genes for cardiac troponin $\mathrm{T}$ and alpha-tropomyosin in hypertrophic cardiomyopathy. N Engl J Med 332: 1058-1064.

6. Arad M, Penas-Lado M, Monserrat L, Maron BJ, Sherrid M, et al. (2005) Gene mutations in apical hypertrophic cardiomyopathy. Circulation 112: 2805-2811.

7. Gersh BJ, Maron BJ, Bonow Ro, Dearani JA, Fifer MA, et a1. (2011) ACCF/ AHA guideline for the diagnosis and treatment of hypertrophic cardiomyopathy: executive summary: A report of the American College of Cardiology Foundation/ American Heart Association Task Force on Practice Guidelines. Circulation 124: $2761-2796$

8. Morita $\mathrm{H}$ (2013) Human genomics in cardiovascular medicine: implications and perspectives. Circ J 77: 876-885.

9. Chan IS, Ginsburg GS (2011) Personalized medicine: progress and promise. Annu Rev Genomics Hum Genet 12: 217-244. 